\section{Zika virus: designate standardized names}

A rapid response by the publichealth and research communities to infectious viral diseases depends on the reproducible tracking and analysis of pathogen isolates. A standard strainnaming convention for Zika virus sequences is therefore urgently needed. This will ensure that the exchange and interpretation of data is unambiguous in efforts to contain the current outbreak in the tropical Western Hemisphere.

Zika virus strain names for isolates associated with the outbreak are arbitrarily designated as BeH818995, ZikaSPH2015 and BR/949/15, for example. Such names are largely opaque and inconsistent when it comes to context, although some may include useful metadata about isolates. It is impractical to include all relevant metadata in the isolate name, but some consistent information is useful for identifying specific isolates.

Building on conventions in other viral fields, we urge the Zika community to adopt a standard nomenclature for isolate names, specifying the virus type (ZIKV), host species abbreviation, geographical location of isolation, unique identification string and year of isolation. The preferred isolate name for BeH818995, for example, would then be ZIKV/H. sapiens/Brazil/ BeH818995/2015.

Richard H. Scheuermann* J. Craig Venter Institute, La Jolla, California, USA. rscheuermann@jcvi.org ${ }^{*}$ On behalf of the Viral Genome Annotation Standards Working Group (see go.nature.com/i5dewk for full list).

\section{Zika virus: accurate terminology matters}

You describe microcephaly as a "serious congenital malformation", which risks confusing the public and causing needless distress to the families of children with small heads, irrespective of whether these are linked to Zika virus infection (see Nature 530, 5; 2016). In fact, 'microcephaly' simply means a small head and is not necessarily associated with intellectual disability, as is often assumed.

Microcephaly is a feature of hundreds of different conditions, but can also be seen in otherwise normal individuals (P. Merlob et al. J. Med. Genet. 25, 750-753; 1988; S. Ashwal et al. Neurology 73, 887-897; 2009).

This is not mere semantics. Investigations into the proposed link between Zika virus and birth defects (for which there seems to be little evidence at present) will need to include systematic assessment of all the possible causes of microcephaly in children thought to have been affected by the virus (C. G. Victora et al. Lancet 387, 621-624; 2016).

Edwin P. Kirk Sydney Children's Hospital; University of New South Wales; and SEALS Laboratories, Randwick, Australia. edwin.kirk@health.nsw.gov.au

\section{How to engage social scientists in IPBES}

We contend that the disciplinary imbalance within the

Intergovernmental Platform on Biodiversity and Ecosystem Services (IPBES) could best be remedied by improving the organization's communication with researchers from the social sciences and humanities (see A. B. M. Vadrot et al. Nature 530, 160; 2016).

Our analysis of the groups that were nominated and selected after the second IPBES call for experts for deliverables 2(b) and 3(b)(i) - namely the regional/subregional assessments of biodiversity and ecosystem services, and of land degradation and restoration indicated that most people who applied for the assessments had a background in natural sciences (see go.nature.com/ pexril). This suggests that IPBES communications about the details and implications of the IPBES process itself might not be effectively engaging the social-science and humanities communities.

We suggest that IPBES calls need to be circulated more widely and avoid language and expressions that are tailored specifically for natural scientists. The calls should recognize differences in the social-science and humanities communities and target these more specifically. Katrin Reuter, Malte Timpte Museum für Naturkunde, Leibniz Institute for Evolution and Biodiversity Science, Berlin, Germany.

Carsten Nesshöver Helmholtz Centre for Environmental Research - UfZ, Leipzig, Germany. malte.timpte@mfn-berlin.de

\section{Better management of alien species}

In our view, the European Union's recent legislation on invasive alien species will be an effective conservation tool only if the inclusion of new species is supported by the majority of EU states. We call for Europe to put the protection of its biodiversity before the short-term economic interests of member states.

Europe is one of the world's most biologically invaded regions (M. van Kleunen et al. Nature 525, 100-103; 2015). But the list of invasive alien species targeted for action under the January 2015 EU legislation includes just 37 entries (see go.nature.com/gigftz) - even though Europe hosts more than 1,000 such species, most of which meet the criteria for listing (M. Vilà et al. Front. Ecol. Envir. 8, 135-144; 2010). For example, knotweed (Fallopia sp.) and American mink (Neovison vison) are well-characterized species that are responsible for extensive biodiversity losses across the continent.
We are concerned that the restricted new listing cannot hope to address the scale of biological invasions in Europe. Management must be coordinated at the EU level if both protective and preventative regulation are to be widely applicable, comprehensive and effective.

Jan Pergl Institute of Botany, The Czech Academy of Sciences, Pruhonice, Czech Republic.

Piero Genovesi Institute for Environmental Protection and Research, Rome, Italy. Petr Pyšek Institute of Botany, The Czech Academy of Sciences, Pruhonice; and Charles University in Prague, Czech Republic. jan.pergl@ibot.cas.cz

\section{Class uncorrected errors as misconduct}

Post-publication peer review is becoming increasingly popular, but authors need more incentive to self-correct and amend the scientific record (see D. B. Allison et al. Nature 530, 27-29; 2016). We propose that failure by authors to correct their mistakes should be classified as scientific misconduct. This policy has already been implemented by our institute, and we encourage research institutions and funding bodies to follow suit (see go.nature.com/dgifft).

The responsibility to correct errors lies mainly with the criticized authors. Snubbing criticism by not addressing it promptly runs counter to our fundamental ethos as scientists, and threatens to erode society's trust in the scientific community. Sophien Kamoun, Cyril Zipfel The Sainsbury Laboratory, Norwich, UK. sophien.kamoun@tsl.ac.uk

CONTRIBUTIONS

Correspondence may be sent to correspondence@ nature.com after consulting the guidelines at http:// go.nature.com/cmchno. 